Published by Intellect in the International Journal of Digital Television, Volume 6 Number 2, June 2015

doi: https://doi.org/10.1386/jdtv.6.2.145_1

\title{
Open data and co-production of public value of BBC Backstage
}

Yuwei Lin, University for the Creative Arts

\section{Abstract}

Openly accessible data sets (open data) have been recognized as valuable assets for creating business opportunities, revitalizing innovation and transparentizing organizational conducts. Public Service Broadcasters (PSB) such as the British Broadcasting Corporation (BBC) have been motivated to experiment with open data and new forms of innovation in content making, delivery and audience engagement. Through a case study of the BBC Backstage project, this article examines how such open innovation processes of engaging the public in the reuse and remix of open data were conceived, supported, managed and maintained. The research found that BBC Backstage had played an important role in encouraging and motivating people to reuse and repurpose the open data released by the BBC. New forms of outputs have emerged, as seen in the Data Arts visualization project and the R\&DTV clips mashups. The article argues that PSB public value can be co-produced through opening up data sets, encouraging reuse and remix, and building up a network of enthusiastic and capable active audiences, the techno-elites, whose status has been encouraged the open data culture and alike. Lessons learned can help understand the meanings of open data from the PSB perspective, and the implications in media industry thereby foster innovation in future media and creative industries.

\section{Keywords}

open data

public value

techno-elite 
Published in the International Journal of Digital Television, Volume 6 Number 2, June 2015 doi: 10.1386/jdtv.6.2.145_1

$\mathrm{BBC}$

co-production

audience participation

\section{Introduction}

With the proliferation of computers, data are being generated and processed at an unprecedented pace and volume (Kitchin and Lauriault 2014). Data-mining techniques have been developed to process different types of data, including transactional data automatically generated by machines, content generated by users on the Internet and digitized old (historical) records. These data are available in different formats, platforms and un/available under different intellectual property rights. Industries, governments and the third sector all have shown keen interests in exploiting the 'big data', referred to 'extremely large data sets that may be analysed computationally to reveal patterns, trends and associations, particularly in relation to human behaviour and interactions' (see the House of Lords 2014: 21; see also Oxford English Dictionary 2013).

Common data practices in computing and the web industry, such as data visualization, mashups of archival content, data mining and machine learning, have become popular practices and adopted in the media industry to engage audiences and to generate, represent and deliver content on different platforms. For example, in data journalism, visual methods such as infographics, videos and interactive web interfaces have been adopted to reveal and represent data, to transparentize the data sources that make news stories more reliable and trustful. Many commercial activities are data driven and algorithmically directed. Tesco Clubcard, Amazon Cloud and Facebook and Google marketing and advertising are well-known examples. In the TV industry, programme making can follow such data-centric methods. 
Published in the International Journal of Digital Television, Volume 6 Number 2, June 2015 doi: 10.1386/jdtv.6.2.145_1

The concept of 'open data' shares a similar philosophy seen in free/libre open-source software (FLOSS) (Stallman 1985), open content and free culture (Lessig 2004). The value of open data has been favourably mentioned in public policy documents and linked with the concept of 'open government'. For example, in the open letter that the UK prime minister David Cameron wrote on 1 June 2010, a timetable for opening up governmental data sets and greater transparency across the UK government was set out so as to

enable the public to hold politicians and public bodies to account; to reduce the deficit and deliver better value for money in public spending; and to realise significant economic benefits by enabling businesses and non-profit organisations to build innovative applications and websites using public data. (Prime Minister's Office 2010)

This statement entails a range of values of open data: democratic and political values of open data to promote participation, support active and informed citizenship (transparency, accountability to citizens), cultural value, educational value, social and community value, global value and economic value.

Parallel to the growing interest in the public sector, a number of initiatives have been launched by PSB around the world to explore opportunities offered by open data. For example, in terms of usergenerated content, the Australian Broadcasting Corporation (ABC) initiated the ABC Pool, a usercreated content space developed and resourced by the $\mathrm{ABC}$, to enable users to publish their audio, video, photography and writing on a platform and to engage audiences in remixing (Hutchinson 2013). The kind of open data that the BBC set off to explore, however, is rather different. BBC Backstage, a pioneering project launched by the BBC Research and Development (R\&D) 
Published in the International Journal of Digital Television, Volume 6 Number 2, June 2015 doi: 10.1386/jdtv.6.2.145_1

department in 2005, was to experiment with how to engage 'the public' in the reuse, remix and repurpose of open data.

This article investigates the impacts of open data on programme making and content production in the convergent media industry, and how it is shaping the concept of 'public value' of public service broadcasting (PSB). I argue that the availability of open data mobilizes co-production and thereby motivates open innovation. PSB public value therefore is not delivered solely by the broadcasters, but co-produced by the PSB and the public. I also argue that a new group of elite audience, the 'techno-elites', has emerged in an age of convergent media because of their ability to manipulate data and software. I use a BBC R\&D project, BBC Backstage, as an example, to illustrate the potential of open data and the opportunities that these techno-elites envision for revolutionizing media content production, provision and distribution. I also discuss the challenges encountered during the operation period of BBC Backstage. I conclude by discussing data-intensive and datadriven content making, programming, commissioning and delivery. Several theoretical concepts such as 'media power', 'active audience', 'elite class' and 'participatory media' are helpful for conceptualizing the sociocultural and socio-technical changes in industry and in a society influenced by open data.

\section{PSB and public value}

The 'public value test' has been introduced as a method to measure the impact of PSB and justify their funding. But what is 'public value'? Some have referred to it as a principle shaping and informing 'institutional governance'. An institution following public value ensues a form of network governance that is self-organizing, collaborative governance or what Mark Moore calls 'co-production' (1995: 117-18), in which users and providers collaborate cooperatively to make 
Published in the International Journal of Digital Television, Volume 6 Number 2, June 2015 doi: 10.1386/jdtv.6.2.145_1

public institutions work. Because they co-produce, users play an active, productive and constitutive role as citizens in public service production and delivery.

Kelly and Muers (2002) emphasize two properties of public value: co-production (involvement of users) and contestation (competition) as the means through which public value can be realized. The concept of 'co-production' is particularly relevant in the context of open data, and highlights the role of audience. As Collins (2007) argues, by extending Moore (1995)'s research in New Public Management (NPM) to the context of PSB,

Public value, with its ethos of co-production - that is partnership between users and providers, potentially combines downward accountability to users, but to users as citizens rather than as subjects or consumers. ... Instead of ratings, public value informed broadcasting would foster cultured and knowledgeable viewers and listeners. Instead of using market mechanisms and price, it would redress market failures and focus on providing the goods and services which either cannot satisfactorily be priced or which should not be provided through price and market regimes. (Collins 2007: 7)

The BBC's answer to the question 'What is public value?' is embodied in the BBC's adoption of five outward looking public purposes (serving citizenship and civil society, promoting education and learning, stimulating creativity and cultural excellence, reflecting the United Kingdom, its Nations, regions and communities and bringing the world to the United Kingdom and the United Kingdom to the world), and the embedding of four 'drivers' (reach, quality, impact and value for money in BBC's operating procedures) (BBC 2004).

In this article, I draw on Moore's articulation of public value (1995), which foregrounds the values 
Published in the International Journal of Digital Television, Volume 6 Number 2, June 2015 doi: 10.1386/jdtv.6.2.145_1

of 'co-production and contestation' to understand the public value of open data. The concepts of 'co-production' and 'contestation' are linked with the three advantages that open data are known for: promoting transparency, releasing social and commercial value and engaging users and audience (Open Knowledge Foundation [OKF] 2012), as discussed below.

\section{Open data}

In the Open Data Handbook Documentation published by the Open Knowledge Foundation (OKF) in 2012, 'openness' can be characterized by three features:

1. Availability and access: the data must be available as a whole and at no more than a reasonable reproduction cost, preferably by downloading over the Internet. The data must also be available in a convenient and modifiable form.

2. Reuse and redistribution: the data must be provided under terms that permit reuse and redistribution including intermixing with other data sets. The data must be machinereadable.

3. Universal participation: everyone must be able to use, reuse and redistribute - there should be no discrimination against fields of endeavour or against persons or groups. For example, 'non-commercial' restrictions that would prevent 'commercial' use, or restrictions of use for certain purposes (e.g. only in education), are not allowed.

In a nutshell, 'open data' refer to any content, information or data that people are free to use, reuse and redistribute - without any legal, technological or social restriction. Whilst some consider anything available, scrubable, aggregatable on the Internet as 'open data', this article adopts a less dubious definition referring to 'open data' as those released under an 'open data licence' or alike, 
Published in the International Journal of Digital Television, Volume 6 Number 2, June 2015 doi: $10.1386 / j d t v \cdot 6.2 \cdot 145 \_1$

which can be freely used, reused and redistributed by anyone.

Open data are praised for the following three reasons (OKF 2012): promoting transparency, releasing social and commercial value, and engaging users and audience. Each of these three advantages is closely linked to the concept of 'public value' both in terms of co-production and contestation, as mentioned in the previous section. Transparency is not only about access; it is also about sharing and reusing. Often, to understand material it needs to be analysed and visualized and this requires the material to be open so that it can be freely used and reused (cf. co-production and contestation). Making data sets openly accessible can help organizations drive the creation of innovative business and services that deliver social and commercial value. Opening up data sets also encourages participatory governance that is essential in a democratic society, or for business and organizations to engage users and audience. In the context of open governments, citizens are enabled to be much more directly informed and involved in decision-making. According to OKF (2012), 'this is more than transparency: it's about making a full "read/write" society, not just about knowing what is happening in the process of governance but being able to contribute to it'. This idea of participation is parallel to the discussion of 'participatory audience' (Carpentier 2003, 2011) or 'active audience' (Morley 1993; Livingstone and Lunt 1994). But, as we will see in the case of $\mathrm{BBC}$ Backstage, the level of participation in reusing open data has been intensified because the audience is involved in the actual making of media products, which requires a level of engagement deeper than call-in, voting or second-screen.

So, how can PSB (re)generate public value in an age of media convergence, where broadcasting goes hand in hand with the Internet, and new content and formats across multiple platforms arise? In the following, I use BBC Backstage as an example to delineate how public value of open data are being realized collectively, specifically how innovation in digital content is being encouraged and 
Published in the International Journal of Digital Television, Volume 6 Number 2, June 2015 doi: 10.1386/jdtv.6.2.145_1

motivated through opening up data sets.

\section{Case study on BBC Backstage}

\section{Research methods}

Backstage was initiated by the BBC R\&D department in 2005. It was firstly introduced at the Open Tech 2005 event (2005), a genre of low-cost, one-day conferences where people experience technologies from 'open source'-style ways of working to repurposing everyday electronics hardware. The intention was to 'radically open up the BBC, publishing information and data feeds, connecting people both inside and outside the organisation, and building a developer community' (BBC Backstage 2010: 2). Backstage, heralded by the mantra 'use our stuff to make your stuff', had made content and data feeds available for people to build upon under a non-commercial basis. Types of open data vary, including subtitles, news, statistical data, traffic updates and data collected in different fields (cultural, scientific, financial, statistical, weather, environmental, transport) that have potential uses and applications.

To understand why a significant PSB like the BBC started to experiment on open data, and how they went about freeing and exploring the (expected and unexpected) value of BBC data sets, a number of qualitative methods were adopted to study the project BBC Backstage. The methods used include document analysis of BBC Backstage's publications, participatory observations at Backstage events for twelve months, narrative analysis on the mailing list and five formal and informal interviews with Backstage's producer and participants located in the United Kingdom and outside the United Kingdom. One of the two formal interviews, lasting for two hours, was carried out face-to-face with the producer, and the other, lasting for 40 minutes, via Skype with an overseas 
Published in the International Journal of Digital Television, Volume 6 Number 2, June 2015 doi: 10.1386/jdtv.6.2.145_1

community member. Both interviews were recorded. The document analysis allows the researcher to historicize the advent and evolution of the project, while the narrative analysis and participatory observation (both online and offline) enable the mapping of the network of stakeholders (participants, decision-makers), their interests and the common practices and activities.

The end-of-project e-book (BBC Backstage 2010, commissioned by the BBC R\&D department to document the project), the Backstage website (BBC Backstage Website), was treated as BBC's official position. The book offers 'a snapshot of some of the projects and events that Backstage was involved with, and of the legacy that it leaves behind' (BBC Backstage 2010). The website and blog, maintained by the $\mathrm{BBC}$ producers, contained views from $\mathrm{BBC}$ insiders. Communications on the mailing list, open for the general public to subscribe to, are still documenting and facilitating the conversation between participants of Backstage or interested parties, provide narratives about how members from different parts of the world exchange their views, offer mutual help and negotiate meanings. Together with other blogs and tweets by the participants, these data offer insights into the attitudes and practices of the participants (the kind of public/audience the $\mathrm{BBC}$ wished to engage with) towards open data.

\section{Data analysis}

According to the slides presented at Google Developer Day 2007 by Ian Forrester, Senior Producer at BBC Backstage from 2006 until its closure, the establishment of Backstage was motivated by the Governors' response to the Review of the BBC's Online services by Philip Graf, who committed the $\mathrm{BBC}$ to using open standards to allow users to find and repurpose $\mathrm{BBC}$ content in more flexible ways (Graf 2004). It is said that within the BBC there are two views: 'One is the traditional view of centralised websites, and the other is more open, de-centralised and a mess. That is Backstage and 
Published in the International Journal of Digital Television, Volume 6 Number 2, June 2015 doi: $10.1386 / j d t v \cdot 6.2 .145 \_1$

in there, somewhere, is the future of the BBC' (Forrester 2007).

The data collected for this case study suggest that a substantial part of Backstage's remit was to encourage data reuse, knowledge exchange, social networking, community engagement and outreach. As declared on Backstage's blog, this new venture was to encourage remix, reuse, reappropriation, reorientation of $\mathrm{BBC}$ content and data. It was positioned as 'BBC's early adopter network to encourage participation and support creativity through open innovation'. In addition to identifying what data sets to release, considerable efforts were also invested in getting people to reuse and repurpose the openly available data sets. To do so, Backstage organized a series of events (hack days, unconferences, regular meet-ups with the locals) in several UK cities to engage a community of software and web developers, web designers, digital media professionals, and to encourage knowledge sharing and reuse of BBC content. Online communication tools such as mailing lists and Ideas Store, a webpage that collated ideas submitted by the public, were also launched to establish a sense of community and a network of mutual support.

The demography of the participants in the project, active on the mailing list and present at the local events, featured mainly workers in the ICT and creative industries (e.g. web designers or developers, people working for or with the $\mathrm{BBC}$ ). The reason for this is likely to be that to reuse and remix data, a certain level of IT competency is needed. As discussed below, despite commonly framed as 'resources that everybody can have access to', to reuse and remix open data does require a certain level of digital media literacy that is higher than usual standards. While 'there's no restriction - open data are open to anyone', the level of data literacy, experiences and skills of manipulating tools for mashups and reuse are still essential. It may be true that the best hacks tend to come from teams that combine different talents and perspectives, but it would not be possible without facilitation and a clear understanding of basic data skills. As a result, the participants in this 
Published in the International Journal of Digital Television, Volume 6 Number 2, June 2015 doi: 10.1386/jdtv.6.2.145_1

project were 'early adopters' of BBC's innovative idea of engaging audiences in 'open innovation'. They were a 'techno-elite' audience who were able to take part in the co-production of convergent media content. This highlights one of the barriers identified for the general public to participate in Backstage activities and for managing, maintaining and sustaining such a collaborative platform. The concept 'techno-elite' has been coined (Lin 2012; Beyl and Lin 2014) particularly to politicize and discuss the implications of such elite-centric phenomena, which is distinct from other participatory audience cultures.

\section{BBC Backstage's innovative outputs}

The open data that the $\mathrm{BBC}$ released included the materials from the $\mathrm{BBC}$ Archive, clips or born digital transactional data or metadata generated by the broadcaster. It was said that over 500 prototypes were envisioned and produced during the project (BBC Backstage 2010: 2). To engage a community of digital artists, web developers and software engineers, Backstage (co-)organized a series of social gatherings (including the highlight 'BBC Hack Day' in 2007) across the country. The format of these events was innovatively organic, usually in an informal, improvisational, open manner (which is unusual), allowing participants to collaborate, interact, exchange knowledge, skills and information, as seen in many Linux User Groups meet-ups (Huysman and Lin 2005). For example, conference programmes are not created beforehand by a programme committee but usually created on the first day with the contributions of all the participants against what they would like to share and learn. Such less prescribed structure invites open innovation. The conception of fancy terms such as 'hack days', 'hackathons' (Leckart 2012), 'unconferences', 'barcamps', 'GeekUp', 'Social Media Cafe', 'Open Cafe' also suggests an innovation in outreach language, and denotes a desire of engaging a social group who is familiar with the workplace culture commonly 
Published in the International Journal of Digital Television, Volume 6 Number 2, June 2015 doi: $10.1386 / j d t v \cdot 6.2 \cdot 145 \_1$

observed at IT companies such as Google or Yahoo (Stewart 2013). On some occasions, BBC Backstage's Hack Day also coincided with other industry events, such as Yahoo's Open Hack, so as to engage with the Yahoo! Developer network. In addition, the key project members also participated in local gatherings of digital media workers, such as the regular Manchester Social Media Cafes, which were sometimes held at the ex- BBC Manchester's building on Oxford Road. Such kind of events and indeed physical milieus were important for Backstage to expand its audience base. IAfter all, the project itself was first launched at OpenTech 2005, which bore much resemblance to these events. In addition to encouraging collaboration, hosting or sponsoring these events also gave the project publicity and helped 'getting the data out there'.

As mentioned earlier, the majority of the participants at these hack events were young males working in ICT or media industries, who have access to the latest technical knowledge and trends on the Internet, and were heavily engaged with Internet cultures. They were equipped with abilities of programming/coding, laptops, smart phones or high-end digital devices - as evidenced by a photo by Darra on Flickr (Darra 2007), the blog by participants Premasagar (Premasagar 2007) and M@(M@ 2007), and an article in The Guardian about the Hack Day 2007 (Kiss 2011). The Hack Day, as the participant M@ put it, 'is a 36-hour marathon of coding, pizza, beer and music sponsored by Yahoo! and the BBC'.

The types of open data used varied including traffic data, weather data, subtitle data, other cultural data (e.g. data about cultural works and artefacts - for example titles and authors). These data could potentially be linked with other open data (e.g. scientific data, financial data (e.g., government accounts (expenditure and revenue) and information on financial markets (stocks, shares, bonds etc.), national or international statistics (such as the census and key socio-economic indicators), environmental data (e.g., data related to the natural environment such as presence and level of 
Published in the International Journal of Digital Television, Volume 6 Number 2, June 2015 doi: 10.1386/jdtv.6.2.145_1

pollutants, the quality and rivers and seas) and transport data (such as timetables, routes, on-time statistics). Through data visualization and montage methods, these data were mashed up, and represented to reveal different meanings or reused for different purposes (e.g., visualizing news content on a map, comparing what the audience was reading on BBC's news website and what the $\mathrm{BBC}$ would like the audience to read, creating a search engine to locate or identify traffic information).

The kinds of data that BBC Backstage published were not completely open either. Some of them were licensed under restricted access and reuse due to the provenance or source of the data. Another barrier identified was this legal restriction; the aspiration of building up an open culture and ecosystem was hindered by IPRs and copyrights. Some of the prominent prototypes that had been developed at these 'hack days' were steered into two funded projects and continued after the closure of the Backstage project: the DataArt (2009-11) and the Channelography (2010-11).

The DataArt project, a 22-month collaboration between BBC Learning Innovation and the Centre for Research in Education, Art and Media (CREAM) at the University of Westminster, funded by the UK Arts and Humanities Research Council (AHRC) and starting from 1 November 2009, collected many of the infographic prototypes that visualized BBC-sourced data or content. It echoed a trend in the convergent media world where data and information are processed and (re)presented in various forms such as diagrams, graphs and maps to facilitate and mediate understanding of (a usually massive amount of) data and information. A classic BBC infographic visualized BBC news data feeds on a rotating globe. As declared on the DataArt's webpage:

$\mathrm{BBC}$ Learning recognises that the interpretation of this data is an increasingly important skill for us all and in this spirit the BBC DataArt project provides public access to 
Published in the International Journal of Digital Television, Volume 6 Number 2, June 2015 doi: 10.1386/jdtv.6.2.145_1

interactive data visualizations of the BBC's online resources be they news information from around the world, web articles, music data or video. We will focus principally on BBC web sources but we will also be cross-referencing with other publicly available data. (n.d., para. 2)

To engage the interested parties (the general public as the team assumed), the project offers

- access to visualizations of BBC information resources and provides commentary text and video material that show (webpage) visitors how to use, interpret and explore them

- learning resources for visitors to find out more about the discipline of visualization, its history and other useful research resources

- a collection of toolkits (tools, tutorials, computer code and access to copyright free data sources people can download and modify) for 'more experienced practitioners' (usually developers or digital artists) to exploit.

Apart from their project webpage and blog, the team also used Facebook to publicize their work and materials (see https://www.facebook.com/pages/DataArt-BBC-Backstage/108238225894676).

The Channelography project, or BBC Dashboard as it was later known, was also a spin-off from the Backstage prototypes. The BBC Dashboard was a daily view of television data collected via the Channelography project. It revealed a few key pieces of data, making them readable 'at a glance', in the manner of a car dashboard or office notice board. Data points included the daily level of repeated programming by channel, the number of films being broadcast and some of the frequently 
Published in the International Journal of Digital Television, Volume 6 Number 2, June 2015 doi: 10.1386/jdtv.6.2.145_1

used terms currently being mentioned in the news and in documentaries. The project was a commission from BBC R\&D, and was designed to explore how detailed data could be aggregated upwards into a consumable, single-screen front end. The project has now been retired as the idea has been integrated into BBC's running architecture.

The Backstage's shot-for-web R\&DTV - http://ftp.kw.bbc.co.uk/backstage/index.whtml - also inspired an award-winning BBC documentary Virtual Revolution (2010). On its website, it is said that

R\&DTV is a monthly technology programme made up of interviews from knowledgeable BBC developers, BBC project experts and experts from around the world. The show came in 3 forms: 1) A brief 5-minute video, containing all the very best bits 2) A longer 30-minute video, containing deeper conversations 3) The Asset Bundle, containing everything we used and didn't use to make the videos above. The clips in the asset bundle were raw straight from the team's cameras and although this may be too much for most people, they were great footage for those who want to remix and mashup our footage with their own or others.

Indeed, the entire R\&DTV project was a strategic move of Backstage, specifically created to address the copyright issues emerged from the participants' needs for remixing and repurposing BBC content. R\&DTV's clips were released under a Creative Commons non-commercial attribution version 2 licence (CC BY-NC-ND 2.0) so that users can 'watch, rip, redistribute and remix' all the contents of Asset Bundle. The Backstage team also hoped the users could contribute back: 'We're pretty excited and ask you to please tell us what you do end up doing with the asset bundle, so we can learn what works and what does not work and fix it next time we release another asset bundle'. 
Published in the International Journal of Digital Television, Volume 6 Number 2, June 2015 doi: 10.1386/jdtv.6.2.145_1

This quote echoes the ethics and practices as observed in the free/libre open source software world (see Feller et al. 2005; Benkler 2006; Amant et al. 2007). Ensued by the BBC R\&DTV, the Virtual Revolution documentary also released some unedited professionally filmed footage from the series for audience to use and make their own documentary. These clips included interviews, aerial shots, graphics and music. Audience can download and reuse / remix them for free under BBC's Digital Revolution Licence (terms and conditions similar to CC BY-NC-ND 2.0) specifically drawn to reflect BBC's non-commercial nature.

\section{Co-producing public value, but by whom?}

From the DataArt project that showed how BBC data could be visualized in a creative and informative way, to the BBC R\&DTV that produced and made a series of raw video and audio footage available for remix, BBC Backstage illustrated some of the best scenarios where the audience is proactively participating in the production, value-adding, innovation process. As recognized in the end-of-project book, Backstage has

changed the way people think, the way the BBC interacted with external designers and developers, and the way that they worked together... It isn't just a few data feeds and some blog posts. Backstage brought about permanent change, for the people who worked there, for its community of external developers and for the BBC. (2010: 2)

Backstage arrived at a time when BBC was drawing up its new agenda around public value (BBC 2004; Anon. 2004; BBC Press Office 2004; Thompson 2004). The publication Building Public Value: renewing the BBC for a digital world (BBC 2004) was considered as the BBC's contribution to the DCMS consultation and debate on Charter Renewal in 2006. In this document, it set out an 
Published in the International Journal of Digital Television, Volume 6 Number 2, June 2015 doi: 10.1386/jdtv.6.2.145_1

agenda of empowering modern audience who is more in control of how, when and where they access the content they like and value. 'While building platforms and better access to more affordable digital technologies is a first step to a fully digital Britain, the second is about opening up the creative potential and public, as well as private, value' (Anon. 2004). The then Director-General Mark Thompson affirmed:

We look forward to a future where the public have access to a treasure house of digital content; a store of value which spans media and platforms, develops and grows over time, which the public own and can freely use in perpetuity. When the traditional one-way traffic from broadcaster to consumer evolves into a true creative dialogue in which the public are not passive audiences but active, inspired participants. (2004)

Creating public value is at the heart of this $\mathrm{BBC}$ document as two of the nine points in the manifesto are about public value: 'Programmes and services that build public value through: active and informed citizenship; British culture and creativity; a revolution in learning; connected communities and building the UK's voice in the world, and "a new test of public value"". It was announced that a public value test would be applied to all new services, and changes to existing services, before approval. A set of measures, both quantitative and qualitative, would be used by the Governors including reach, quality, impact and value for money, and the economic value and potential market impact for the test. The BBC would also commission an independent Public Value Survey of 10,000 people every three to five years.

The creation of Backstage in this context symbolizes BBC's willingness and commitment to experimenting how to engage with audience in digital/technical communities by transparentizing and opening up BBC's data feeds and content archives. Allowing audience to reinterpret, play, 
Published in the International Journal of Digital Television, Volume 6 Number 2, June 2015 doi: 10.1386/jdtv.6.2.145_1

reappropriate and remake BBC's data seemed to empower the audience and enlarge BBC's public value. Public value could be diversified, measured and approached from different angles in different ways to include a wide range of innovative, technical, artistic, cultural and economic value through creating added value services by remixing and repurposing data and content.

Other documents also suggested that Backstage was significant in terms of the BBC's online strategy. Documented in the end-of-project e-book, the producer Ian Forrester said that

Backstage marked the point when the BBC started to take online seriously. Although it had an extensive web presence in 2005, especially around news, there was no real sense of any radical agenda behind the corporation's online presence. (BBC Backstage 2010: 6)

As noted in the closing-down statement by the head of BBC R\&D, who oversaw the BBC Backstage project,

BBC Backstage has been a great success.... [T] he BBC recognises that the success of Backstage is down to the whole community of developers, designers, contributors (and even critics) built around the project - a community who pushed it far beyond its original concept. (Woolard 2010)

These statements highlighted that the public value of open data and can therefore be seen as coproduced and co-realized through audience participation. This echoes the kind of open innovation that von Hippel depicts (2005).With open data, the process of content-making was democratized people who have the capabilities can produce content based on the open data. 
Published in the International Journal of Digital Television, Volume 6 Number 2, June 2015 doi: 10.1386/jdtv.6.2.145_1

However, the case study has also shown that those who were involved in data reuse and knowledge sharing were a capable, technically savvy 'audience' who were also keen on exploring the power of open data. The initial idea of Backstage was to 'get the BBC's data out'. But later on, under different leaderships, it has been developed into a (cross-industry / cross-community) developer/designer network from the $\mathrm{BBC}$, an opportunity for the $\mathrm{BBC}$ to offer some of the content and services, a way to share with third party, non-commercial developers, and BBC's way of stimulating creativity and innovation in the market. Some participated for fun and others for commercial purposes.

As seen from the DataArt and the Channelography projects, data (in different forms and formats) are considered the key to future media production. As one can see from BBC's job vacancies, data analysts, people with abilities to manipulate and analyse and present data, are much sought after these days. Audience engagement or audience analysis is now done behind the scene based on data, whether these are transactional data, such as the number of views on iPlayer or traditional TV show ratings, or audience-generated data, such as Facebook messages, tweets or posts on the BBC's Points of View's message boards.

The prototypes and ideas shared on Backstage's webpage reveal that the most used data are those in XML formats: such as travel, news and weather data. In a developer-led environment these data can be (semi-)automatically scraped, aggregated, processed, presented/visualized at speed at large scale. Unlike the kind of user-generated content seen on the ABC Pool, the kind of open data BBC Backstage released and the kind of prototypes it focused on developing were tailored for the developers' community (e.g., computer programmers and others involved in software and hardware development, including graphic designers, game developers, web developers), who did not represent the ordinary mass audience. This can be observed by the outreach language employed and 
Published in the International Journal of Digital Television, Volume 6 Number 2, June 2015 doi: 10.1386/jdtv.6.2.145_1

the channels used to engage and publicize the events (e.g., 'unconferences', 'hackathons'). Many prototypes applied machine-learning or data-mining techniques on BBC's textual data, seeking patterns in the data through clustering, text categorization, topic recognition, demographic/gender/age identification, trend identification and tracking, time series forecasting, measuring predictability of phenomena. Again, these creations and the adoption of AI techniques could be linked with this specific group of audience's everyday practices in software development.

BBC Backstage has brought the notion of 'participatory audiences' (Jenkins 2006) to another dimension through meaningful participation that Carpentier (2011) notes. Audience, in this case, does not just call-in (Munson 1993; Livingstone and Lunt 1994; Shen 1999) or second-screen, waiting for their messages to be selected by media professionals; instead, they take initiative to utilize new technologies, choose useful and/or usable BBC content and data, create extra value out of them and republish and redistribute them on other media platforms. Through reusing, repurposing and reappropriating media content or data, the techno-elite generates new products and value that potentially contribute towards a greater creative economy. The kinds of participatory activities, driven by data associated with Backstage therefore, are not the same as others observed on the social media: redistributing, recirculating, commenting on current affairs. It would seem that these data-embedded participatory activities are more sophisticated, more elitist and less populist.

Because of the participants' active participation, many challenges (such as the limitations of copyrights, needs for Application Programming Interfaces [APIs] and good-quality data, and BBC's relatively bureaucratic institutional policies) were identified and addressed to a certain extent. The kind of data available and strategies adopted have limited the variety of innovation. Certain types of prototypes were favoured (e.g., the Data Arts visualization). The intervention of the BBC has directed the BBC Backstage project to enter a more linear path in relation to open data. As a result, 
Published in the International Journal of Digital Television, Volume 6 Number 2, June 2015 doi: 10.1386/jdtv.6.2.145_1

even if certain innovations have emerged from publicizing open data sets, it is difficult to suggest to what extent socio-economic value can be generated.

\section{Media power and techno-elites}

Media power, in light of Bourdieu (1991) and Couldry (2000), is normally seen as 'concentrated in media institutions of the symbolic power of "constructing reality" (both factual representations and credible fictions)' (Couldry 2000: 4). It is in a transitional process to be divided, shared, negotiated between audiences and producers. Freedman paraphrases Couldry's idea well:

media power is not a tangible object, possessed by institutions and circulated to beguiled audiences but a social process organized on the basis of a constantly renewed distinction between a manufactured (and rather dazzling) 'media world' and the 'ordinary world' of non-media people. (Freedman 2014: 14)

How media power is fractured and decentralized by the development and the adoption of digital technologies has been discussed extensively in scholarly publications. For example, Jeff Jarvis describes this as a 'power shift' (2009: 11) in which 'the shift from mass [to niche] is really a shift of power from top to bottom, center to edge, them to us' (2009: 67). In their description of 'Wikinomics', Tapscott and Williams suggest that digital 'weapons of mass collaboration' are 'ushering us toward a world where knowledge, power, and productive capability will be more dispersed than at any time in our history' (2008: 12). For Henry Jenkins, the process of convergence makes it much harder for elites to impose their authority. He contrasts 'old media's 'power to marginalize' with today's bottom-up 'power to negate' (2006: 278) that is facilitated by new peerto-peer networks, characterized by 'prosumption' and underpinned by a collapse of deference. 
Published in the International Journal of Digital Television, Volume 6 Number 2, June 2015 doi: 10.1386/jdtv.6.2.145_1

'Democracy has always been a messy business' he argues, but today's 'politics of parody', in which citizens use digital tools in creative and autonomous ways to express their cynicism towards 'official politics', is a perfectly logical response to the changing dynamics of authority (2006: 293). For Freedman, 'the most dramatic illustration of this view of dispersed media power - and of the interconnections between political, economic and technological contexts - is to be found in claims made about the 'revolutionary' role of social media in the Arab Spring of 2011 and the possibilities for networked protest (Castells 2011; Mason 2012)' (Freedman 2014: 21).

While such a populist perspective has been celebrated in recent literature, the findings of the study of BBC Backstage suggest that the audience group is not homogeneously capable of nor interested in manipulating open data. The kind of traditional elites whom Jenkins (2006) refers to may be starting to fall out of the media power picture, a new class of elite, the techno-elite, whose position is more versatile and more dynamic is emerging. The population of these early adopters participating in the BBC Backstage's events was those 'techno elites', who potentially have power to challenge, recreate or reclaim authoritative identity. Their prosumptive actions and activities shape the authoritative nature of content author-reader/producer-consumer relationships (Lin 2012; Beyl and Lin 2014).

'Techno-elites' enter the field and negotiate media power. Unlike traditional elite groups who are defined in terms of income or occupation or some other clearly indicative index (e.g., lawyers, bankers, politicians, aristocrats), 'techno-elites' are powerful because of their abilities to make, translate or distribute messages across digital devices and platforms. Being able to operate crossplatform has significant implications in an age of convergent media. Data, information and knowledge can be consumed by more people and in different ways. The focus on abilities and knowledge liberates the definition of 'elite', and places an emphasis on producing an elite class 
Published in the International Journal of Digital Television, Volume 6 Number 2, June 2015 doi: 10.1386/jdtv.6.2.145_1

through education, inclusiveness, possibility of broadening access to resources and socio-economic and socio-technical changes.

Freeing up the data sets would invite more local practices (Couldry 2000: 155) and situated interpretations as they mobilize

the interaction of institutional structures, modes of representation, access to media technologies and broader issues of political authority and civic engagement, framed by an interest, in relation to an earlier point, with the location rather than the allocation, of media power. (Freedman 2014: 14)

Techno-elite audience plays a key role in this dynamic power relationship, occupying different social worlds (commercial media industry or IT industry, civic community). Since the class is not defined by fixed labels or properties, but a set of skills, knowledge and practices, the emergence of the techno-elite audience mirrors the changing society structure and hierarchy, and the expansion of IT companies in an age of media convergence. The concept of 'techno-elite' not only politicizes the participatory media landscape but also provokes thoughts on how to increase the 'capitals' that people need to become a techno-elite. Would improved media or digital literacy help with the recruitment of techno-elite? Would the growth of techno-elite lead to an increase of public value of open data?

In terms of elite recruitment, it is sensible to think that increasingly more audiences will be moving to the category of 'techno-elite' and many traditional middle class can also occupy this position so long as they have access to technologies and possess capabilities of engaging in a repertoire of shared practices (and it is likely that the already powerful elite will be given even more power when 
Published in the International Journal of Digital Television, Volume 6 Number 2, June 2015 doi: 10.1386/jdtv.6.2.145_1

they occupy an overlapping category of techno-elite). Potentially, 'the public' should be trained to be 'techno-elites', to be able to creatively and critically engage in the open data movement. The public value of open data should therefore be considered to be co-produced by the BBC with the techno-elite, the audience and the public.

\section{Conclusions}

The article argues that PSB public value can be co-produced through such open innovation methods, freeing data sets, encouraging reuse and remix, and building up a network of enthusiastic and capable 'techno-elite' audiences. Through the case study of BBC Backstage, it has explained how releasing PSB data as open data can (re)generate public value, and how public value of open data can be co-produced by the $\mathrm{BBC}$, the participants who manipulate the open data and other businesses or organizations who are all key stakeholders in the open-data ecosystem. In other words, PSB is not the only actor responsible of the delivery of its own public value once the data are released; the value of open data is fluid and requires currency to facilitate the exchange.

However, this article problematizes the open innovation introduced by BBC Backstage by arguing that a specific group of elite audience - the techno-elites - have been targeted for involvement in this open data movement. This co-production perspective requires a rethink by questioning how media power is being (re)distributed in the exercise of reuse and reappropriation of open data. Taking the usual political question 'values created by whom and for whom' to a different level, enquiring 'values co-produced by whom and for whom' would highlight the civic responsibility and accountability. It also allows us to rethink what kind of media and information literacies contemporary audiences and users should develop in an age of convergent media. 
Published in the International Journal of Digital Television, Volume 6 Number 2, June 2015 doi: $10.1386 / j d t v \cdot 6.2 \cdot 145 \_1$

This case study of BBC Backstage has revealed that public value cannot be considered as a fixed property, nor can it be evaluated by ticking boxes. It would be difficult to measure the five categories that $\mathrm{BBC}$ defines for public value - democratic and civic value, cultural value, educational value, social value and global value - if one does not bring in fluidity of different capitals (in Bourdieu's term) into account. As 'media power', which is symbolic and dynamic, public value should be treated as a continuous process with struggles, interplay and negotiation between different stakeholders in a media ecosystem. Public value, in the case of BBC Backstage, is embodied through mundane practices, reusing, remixing and repurposing open data. Without a predefined set of categories, the concepts of 'co-production' and 'contestation' embedded in public value are not evaluated by indicators but by observing who were participating, and how they were participating. The discussion of 'techno-elite' class in this article is therefore particularly beneficial. This practice-based perspective opens up discussion about diverse meanings and perceptions of 'public value' of public service media in different contexts, and the levels of participation of different audiences (elite or non-elite).

\section{Acknowledgements}

The author would like to thank the guest editor and the two anonymous reviewers for their comments and guidance in helping to improve this article. She would also like to thank the members of the BBC Backstage for sharing their experiences that enrich the article.

\section{References}


Published in the International Journal of Digital Television, Volume 6 Number 2, June 2015 doi: $10.1386 / j d t v \cdot 6.2 .145 \_1$

Amant, Kirk St. and Brian, Still (eds) (2007), Handbook on Research in Open Source Software: Technological, Economic and Social Perspectives, Hershey, PA: Idea Group Inc.

Anon. (2004), 'Building public value: the BBC's submission - the BBC's main proposals in its application for charter renewal', The Guardian, 29 June, http://www.guardian.co.uk/media/2004/jun/29/broadcasting.bbc. Accessed 28 March 2015.

BBC (2004), 'Building public value: Renewing the BBC for a digital world', http://downloads.bbc.co.uk/aboutthebbc/policies/pdf/bpv.pdf . Accessed 28 March 2015. (2010), Virtual Revolution, http://www.bbc.co.uk/programmes/b00n4j0r. Accessed 21 April 2015.

BBC Backstage (2010), 'Hacking the BBC: A backstage retrospective'. London: The BBC.

BBC Backstage Website, http://www.bbc.co.uk/blogs/bbcbackstage. Accessed 27 April 2015 (no longer maintained).

BBC Press Office (2004), 'BBC launches its vision of the future and manifesto for action', 29 June, http://www.bbc.co.uk/pressoffice/pressreleases/stories/2004/06 june/29/bpv.shtml. Accessed 28 March 2015.

Benington, J. (2009), From Private Choice to Public Value. In Public Value: The Theory and Practice (ed. J. Benington and M. Moore), Basingstoke: Palgrave. 
Published in the International Journal of Digital Television, Volume 6 Number 2, June 2015 doi: $10.1386 / j d t v \cdot 6.2 .145 \_1$

Benkler, Y.(2006), The Welath of Networks: How Social Production Transforms Markets and Freedom, London: Yale University Press.

Beyl, J. and Lin, Y.-W. (2014), 'Using linguistic ethnography to study techno-cultural eliteness of social media users', in Frauke Zeller, Cristina Ponte and Brian O’Neill (eds), Revitalising Audiences: Innovations in European Audience Research, London: Routledge, pp. 106-22.

Bourdieu, P. (1991), Language and Symbolic Power, Cambridge: Polity.

Castells, M. (2011), 'A network theory of power', International Journal of Communication, 5, pp. $773-87$.

Carpentier, N. (2003), 'BBC’s Video Nation as a participatory media practice. Signifying everyday life, cultural diversity and participation in an on-line community', International Journal of Cultural Studies, 6(4): 425-447.

Carpentier, N. (2011), 'The concept of participation. If they have access and interact, do they really participate?', CM: Communication Management Quarterly, 21, pp. 13-36.

Channelography, (n.d.), http://rattlecentral.com/channelography/. Accessed 27 April 2015.

Collins, R. (2006), ‘The BBC and public value', CRESC Working Paper no. 19, Centre for Research on Socio-Cultural Change (CRESC), Open University. 
Published in the International Journal of Digital Television, Volume 6 Number 2, June 2015 doi: $10.1386 / j d t v \cdot 6.2 .145 \_1$

(2007), 'Public Value and the BBC'. A report prepared for The Work Foundation's public value consortium, March,

http://www.theworkfoundation.com/assets/docs/publications/174_publicvalue bbc.pdf. Accessed 17 April 2015.

Couldry, N. (2000), The Place of Media Power: Pilgrims and Witnesses of the Media Age, London: Routledge.

Darra, C. (2007), 'bbc yahoo london hackday' 